\title{
Simulation and reversal of $n$-qubit Hamiltonians using Hadamard matrices
}

\author{
Debbie W. Leung \\ IBM TJ Watson Research Center, P.O. Box 218, Yorktown Heights, NY 10598
}

(February 20, 2019)

The ability to simulate one Hamiltonian with another is an important primitive in quantum information processing. In this paper, a simulation method based on Hadamard matrices and applicable for arbitrary $\sigma_{z} \otimes \sigma_{z}$ interaction (quantph/9904100) is generalized for any pairwise interaction. We describe two applications of the generalized framework. First, we obtain a class of protocols for selecting an arbitrary interaction term in an $n$-qubit Hamiltonian, a special case of which is given in quant-ph/0106064 2 . Second, we obtain a class of protocols for inverting an arbitrary, possibly unknown $n$-qubit Hamiltonian, generalizing the result in quant-ph/0106085 1.

\section{INTRODUCTION}

An important element in quantum information processing is the ability to efficiently convert a set of primitives, determined by the physical system, to perform the desired task. In many physical systems, the primitives are "local manipulations" such as fast single qubit operations that can be controlled, and a given nonlocal system Hamiltonian that cannot be changed. In this case, the desired task may be approximated or simulated by interspersing the given Hamiltonian evolution with local manipulations. The resources of simulation include the amount of local manipulations and the total operation time of the given Hamiltonian.

Such simulation was extensively studied in the context of NMR quantum computation [1] 3] in which the naturally occurring Hamiltonian cannot be controlled. Reference [2] presents a method based on Hadamard matrices to convert any given Hamiltonian of the form

$$
H_{1}=\sum_{i<j} g_{i j} \sigma_{z}^{(i)} \otimes \sigma_{z}^{(j)}+\sum_{i} \omega_{i} \sigma_{z}^{(i)}
$$

to a particular term $g_{l m} \sigma_{z}^{(l)} \otimes \sigma_{z}^{(m)}$ where $\sigma_{z}^{(i)}$ is a Pauli matrix acting on the $i$-th qubit. 1 The same protocol applies universally for all coefficients $g_{i j}$ and $\omega_{i}$. Other arbitrary evolutions can in turns be obtained by reduction to the universality construction [4] 6 . The simulation of

${ }^{1}$ A similar method was reported independently in Ref. 3 . the single term is exact, and does not require frequent local manipulations. A related task to stop the interaction is also addressed. The method aims at minimizing the required number of single qubit gates, which is slightly higher than $n^{2}$.

A more general problem was addressed recently in Ref. [7]. A major step is to convert the Hamiltonian

$$
H_{2}=\sum_{i j} \sum_{\alpha \beta} g_{i j \alpha \beta} \sigma_{\alpha}^{(i)} \otimes \sigma_{\beta}^{(j)}+\sum_{i} \vec{r}^{(i)} \cdot \vec{\sigma}^{(i)}
$$

to a single term $g_{l m \gamma \eta} \sigma_{\gamma}^{(l)} \otimes \sigma_{\eta}^{(m)}$, where $\sigma_{\alpha=x, y, z}$ denote the Pauli matrices. The single term is then used to simulate the dynamics due to an arbitrary Hamiltonian $H_{2}^{\prime}$ similar to Eq. (2). For each required accuracy level, both local and nonlocal resources are analyzed.

Related problems were discussed in Refs. 8,99. Bounds are derived for the operation time of the particular Hamiltonian

$$
H_{3}=\sum_{i j} \sigma_{z}^{(i)} \otimes \sigma_{z}^{(j)}
$$

to simulate an arbitrary Hamiltonian given by Eq. (2). Bounds on the operating time are also obtained for simulating the inverse evolution $e^{i H_{4} t}$ of the Hamiltonian ${ }^{2}$

$$
H_{4}=\sum_{\alpha} d_{\alpha} \sum_{i j} \sigma_{\alpha}^{(i)} \otimes \sigma_{\alpha}^{(j)}
$$

which is essentially the most general pairwise interacting Hamiltonian with permutation symmetry. 3

The general principle in these simulation schemes is to transform some coupling terms to the desired form and to cancel out the rest, by interspersing the free evolution with single qubit operations. In this paper, we generalize the framework in Ref. 20 to apply to Hamiltonians of the form Eq. (2). From this framework, we find a class of schemes that selects a term $\sigma_{\gamma}^{(l)} \otimes \sigma_{\eta}^{(m)}$ from a Hamiltonian given by Eq. (2). The method presented

\footnotetext{
${ }^{2}$ Throughout this paper, the time evolution due to a Hamiltonian $H$ is given by $e^{-i H t}$. Note the - sign in the exponent. The inverse evolution is given by $e^{i H t}$. This notation follows from the Schrödinger equation.

${ }^{3}$ The two-qubit case was independently considered in Ref. 110
} 
in Ref. [7] is a particular scheme within the generalized framework. We also find a class of schemes for simulating time reversal for Hamiltonians of the form of Eq. (3) that are very different from those in Ref. 99. Finally, we present a protocol to reverse an arbitrary Hamiltonian given by Eq. (2) that requires operation time $\approx 3 n t$ of $H_{2}$ to simulate the reversed evolution $e^{i H_{2} t}$. The schemes are universal, and apply even when $\mathrm{H}_{2}$ is unknown. This significantly generalizes the results in Ref. 99.

The paper is structured as follows. In Section III, we review the framework and various resulting schemes in Ref. [2], with a slight change from the original NMR based notations. The framework is generalized for any Hamiltonian given by Eq. (2) in Section III. The first application select individual coupling terms from the given Hamiltonian is discussed in Section IV. The second application, simulating time reversal, is discussed in Section $\mathrm{V}$ as a simple application.

\section{SELECTIVE COUPLING USING HADAMARD MATRICES - A REVIEW}

\section{A. Statement of the problem}

We review the method developed in [2]. Consider an $n$-qubit system, evolved according to the Hamiltonian

$$
H_{z}=\sum_{i<j} g_{i j} \sigma_{z}^{(i)} \otimes \sigma_{z}^{(j)}
$$

where $g_{i j}$ are arbitrary coupling constants. The goal is to evolve the system according to only one term of the Hamiltonian:

$$
\sigma_{z}^{(i)} \otimes \sigma_{z}^{(j)}
$$

using single qubit operations. We call this task "selective coupling." This is closely related to the task of stopping the evolution or "decoupling". We first develop a framework for decoupling. Then we construct decoupling and selective coupling schemes using Hadamard matrices.

\section{B. Decoupling scheme for two qubits}

We motivate the general construction using the simplest example of decoupling two qubits. Let $U_{t}=e^{-i H_{z} t}$, where the Hamiltonian is given by $H_{z}=g_{12} t \sigma_{z}^{(1)} \otimes \sigma_{z}^{(2)}$. We use the shorthand $X^{(i)}$ for $\sigma_{x}^{(i)}$. We also use the important identity

$$
U e^{M} U^{\dagger}=e^{U M U^{\dagger}}
$$

where $M$ is any bounded square matrix and $U$ is any unitary matrix of the same dimension. As the Pauli matrices anticommute,

$$
\begin{aligned}
X^{(2)} U_{t} X^{(2)} & =X^{(2)} e^{-i g_{12} t \sigma_{z}^{(1)} \otimes \sigma_{z}^{(2)}} X^{(2)} \\
& =e^{-i g_{12} t \sigma_{z}^{(1)} \otimes\left(X^{(2)} \sigma_{z}^{(2)} X^{(2)}\right)} \\
& =e^{-i g_{12} t \sigma_{z}^{(1)} \otimes\left(-\sigma_{z}^{(2)}\right)}=U_{t}^{-1}
\end{aligned}
$$

Thus adding the gate $X^{(2)}$ before and after the free evolution reverses it, and

$$
X^{(2)} U_{t} X^{(2)} U_{t}=I
$$

This illustrates how single qubit operations can transform the action of one Hamiltonian to another.

Equation (8) can be written to highlight some essential features leading to decoupling:

$$
e^{-i g_{12} t\left(+\sigma_{z}^{(1)}\right) \otimes\left(-\sigma_{z}^{(2)}\right)} \times e^{-i g_{12} t\left(+\sigma_{z}^{(1)}\right) \otimes\left(+\sigma_{z}^{(2)}\right)} .
$$

Each factor corresponds to a "time interval" of evolution. 1. In each interval, each $\sigma_{z}^{(i)}$ acquires a - or + sign, according to whether $X^{(i)}$ are applied or not before and after the time interval.

2. The bilinear coupling is unchanged (negated) when the signs of $\sigma_{z}^{(1)}$ and $\sigma_{z}^{(2)}$ agree (disagree).

3. Since the matrix exponents commute, negating the coupling for exactly half of the total time is necessary and sufficient to cancel out the coupling.

The crucial point leading to decoupling is that, the signs of the $\sigma_{z}$ matrices of the coupled qubits, controlled by the $X$ gates, disagree for half of the total time elapsed.

\section{Sign matrix and decoupling criteria}

We now generalize the framework for decoupling to $n$ qubits. Each of our schemes concatenates some $m$ equaltime intervals. In each time interval, the sign of each $\sigma_{z}^{(i)}$ can be + or - as controlled by the $X$ gates. Each scheme is specified by an $n \times m$ "sign matrix", with the $(i, a)$ entry being the sign of $\sigma_{z}^{(i)}$ in the $a$-th time interval. The entries in each column represent the signs of all the qubits at each time interval and the entries in each row represents the time sequence of signs for each qubit. We denote a sign matrix for $n$ qubits by $S_{n}$. For example, the scheme in Eq. (9) can be represented by the sign matrix

$$
S_{2}=\left[\begin{array}{ll}
+ & + \\
+ & -
\end{array}\right]
$$

Following the discussion in Sec. II B, we have

$$
\begin{aligned}
& \text { Decoupling criteria I Decoupling is } \\
& \text { achieved if any two rows in the sign matrix } \\
& \text { disagree in exactly half of the entries. }
\end{aligned}
$$

For example, the following sign matrix decouples four qubits: 


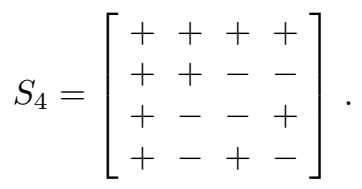

More explicit, the scheme is given by

$$
\begin{aligned}
& U_{t} \times\left(X^{(3)} X^{(4)} U_{t} X^{(3)} X^{(4)}\right) \times \\
& \quad\left(X^{(2)} X^{(3)} U_{t} X^{(2)} X^{(3)}\right) \times\left(X^{(2)} X^{(4)} U_{t} X^{(2)} X^{(4)}\right),
\end{aligned}
$$

where $U_{t}=e^{-i H_{z} t}$ has six possible coupling terms. The relation between the scheme and $S_{4}$ is illustrated in Figure 1.
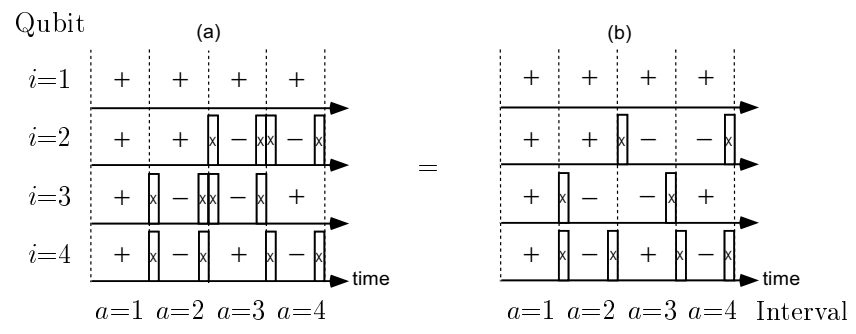

FIG. 1. (a) Converting the sign matrix $S_{4}$ to the scheme in Eq. 12. A "-" sign in the $i$-th row and $a$-th column translates to $X^{(i)}$ (acting on the $i$-th qubit) before and after the $a$-th time interval. (b) Simplifying the scheme using $X^{(i)} X^{(i)}=I$.

From now on, we only consider the sign matrices, which completely represent the corresponding schemes. For $n$ qubits when $n$ is large, $n \times m$ sign matrices with small $m$ can be difficult to construct. For example,

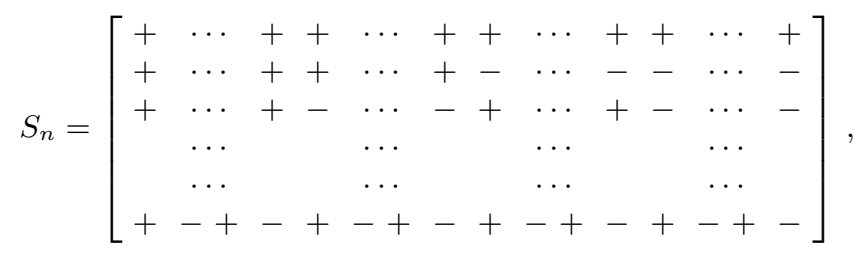

in which an interval from a previous row is bifurcated takes $m=2^{n-1}$. However, the number of columns represents local resources for the simulation. We now resolve this problem, first by rephrasing decoupling criteria I.

Decoupling criteria II Identifying \pm with \pm 1 in $S_{n}$, decoupling is achieved if any two rows having zero inner product, or $S_{n} S_{n}^{T}=$ $n I$.

We now present very efficient solutions to the decoupling criteria, namely the Hadamard matrices [11 14].

\footnotetext{
${ }^{4}$ Note that the commuting factors in Eq. (12) are arranged to visually correspond to the sign matrix.
}

\section{Hadamard matrices and decoupling scheme}

A Hadamard matrix of order $n$, denoted by $H(n)$, is an $n \times n$ matrix with entries \pm 1 , such that

$$
H(n) H(n)^{T}=n I .
$$

Thus every $H(n)$, if exists, is a valid sign matrix corresponding to a decoupling scheme for $n$ qubits using only $n$ time intervals. The following is a list of interesting facts about Hadamard matrices (see Refs. 11 14 for details and proofs).

1. Equivalence Any permutation, or negation of any row or column of a Hadamard matrix preserves the orthogonality condition. Thus each Hadamard matrix can be transformed to a normalized one, which has only +'s in the first row and column.

2. Necessary conditions $H(n)$ exists only for $n=1$, $n=2$ or $n \equiv 0 \bmod 4$.

3. Hadamard's conjecture [15] $H(n)$ exists for every $n \equiv 0 \bmod 4$. This famous conjecture is verified for all $n<428$.

4. Sylvester's construction [16 If $H(n)$ and $H(m)$ exist, then $H(n) \otimes H(m)$ is a possible $H(n m)$. In particular, $H\left(2^{r}\right)$ can be constructed as $H(2)^{\otimes r}$, which is proportional to the matrix representation of the Hadamard transformation for $r$ qubits.

5. Paley's construction [17] Let $q$ be an odd prime power. If $q \equiv 3 \bmod 4$, then $H(q+1)$ exists; if $q \equiv 1 \bmod 4$, then $H(2(q+1))$ exists.

6. Numerical facts [11 For an arbitrary integer $n$, let $\bar{n}$ be the smallest integer satisfying $n \leq \bar{n}$ with known $H(\bar{n})$. For $n \leq 1000, H(n)$ is known for every but 6 possible orders, and $\bar{n}-n \leq 7$. For $n \leq 10000, H(n)$ is unknown for only 192 possible orders and $\bar{n}-n \leq 31$.

The nontrivial existence of so many Hadamard matrices may be better appreciated by examining the following example of $H(12)$, obtained with Paley's construction.

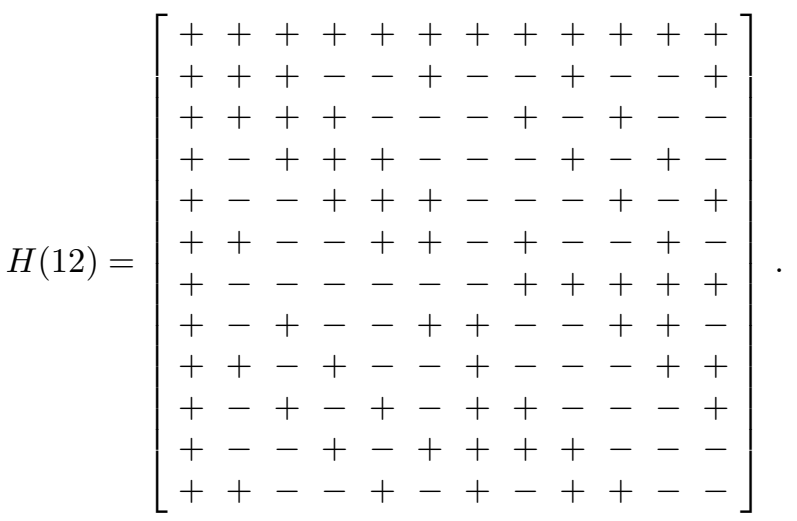


Thus there is a simple decoupling scheme for $n$ qubits if $H(n)$ exists. Using Hadamard matrices, decoupling and recoupling schemes for an arbitrary number of qubits can be easily constructed, as will be shown next.

\section{E. Decoupling and selective coupling}

Decoupling When an $H(n)$ exists, it corresponds to a decoupling scheme for $n$ qubits concatenating only $n$ time intervals. When an $H(n)$ does not necessarily exist, consider $H(\bar{n})$ and choose any $n$ rows to form an $S_{n}$. Then $S_{n}$ corresponds to a decoupling scheme for $n$ qubits requiring $\bar{n}$ time intervals. As an example, $S_{9}$ can be chosen to be the first nine rows of $H(12)$ in Eq. (14):

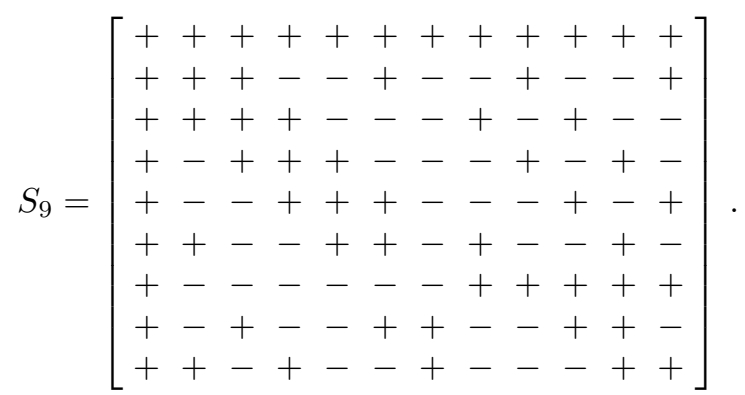

Selective coupling To implement selective coupling between the $i$-th and the $j$-th qubits, any two rows in the sign matrix should be orthogonal, except for the $i$-th and $j$-th rows that are identical. The coupling $g_{i j} \sigma_{z}^{(i)} \otimes \sigma_{\mathrm{z}}^{(j)}$ acts all the time while all other couplings are canceled. The sign matrix can be obtained by taking $n-1$ rows from $H(\overline{n-1})$. For example, to couple the last two among 9 qubits, we can take $S_{8}$ to be the $9 \times 8$ matrix obtained from appending the last row of $H(8)$ to itself. Alternatively, we can take the 2 -nd to the 9 -th rows of $H(12)$ in Eq. (14), and repeat the last row:

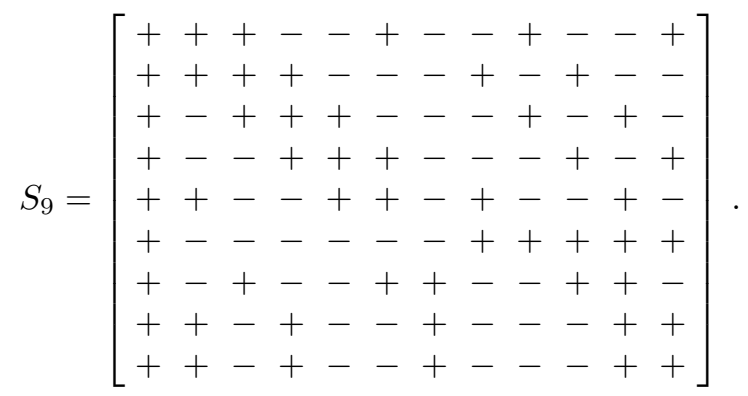

The extra feature of this $S_{9}$ is that, all row sums are zero. This is because $H(12)$ in Eq. (14) is normalized, so that all rows except for the first have zero row sums. This automatically removes any local (linear) terms $\sum_{i} \omega_{i} \sigma_{z}^{(i)}$ in the Hamiltonian, without extra local resources (see Ref. [2] for a full discussion). Finally, we note that coupling terms involving disjoint pairs of qubits can be selected simultaneously.

\section{F. Discussion}

Upper bound on $\bar{n}$ For $n$ qubits, selective coupling requires at most $\bar{n}$ intervals and $n \bar{n}$ single qubit gates. In fact, $\bar{n}=c n$ where $c$ is very close to the idea lower bound $c=1$. First, if Hadamard's conjecture is proven, $\bar{n}$ only depends on $n \bmod 4$, and $\forall_{n} \bar{n}-n \leq 3$. Even without this conjecture, the present knowledge in Hadamard matrices implies $\bar{n}-n \leq 8 \forall n \leq 1000$, and $\bar{n}-n \leq 32 \forall n \leq 10000$. A detailed proof for $c \approx 1$ for arbitrarily large $n$ is given in an Appendix of Ref. [2], while Sylvester's construction puts an immediate loose bound of $c<2$.

Gate simulation vs dynamics simulation 10] The previous discussion assumes that the goal is to simulate the final unitary transformation due to the Hamiltonian $\sigma_{z}^{(i)} \otimes \sigma_{z}^{(j)}$ for time $t$. Due to the commutivity of all the possible coupling terms, we only need to divide the time into $\bar{n}$ time intervals, each with finite duration $t / \bar{n}$. On the other hand, if the goal is to simulate the dynamics due to $\sigma_{z}^{(i)} \otimes \sigma_{z}^{(j)}$ for time $t$, one needs to divide $t$ into small increments $\Delta t$, and to iterate the scheme to simulate $e^{-i \sigma_{z}^{(i)} \otimes \sigma_{z}^{(j)} \Delta t}$ for $t / \Delta t$ times.

\section{GENERALIZED FRAMEWORK FOR ARBITRARY $N$-QUBIT HAMILTONIANS}

We now generalize the previous method for an $n$-qubit Hamiltonian given by Eq. (11) to the more general one given by Eq. (2):

$$
H_{2}=\sum_{i j} \sum_{\alpha \beta} g_{i j \alpha \beta} \sigma_{\alpha}^{(i)} \otimes \sigma_{\beta}^{(j)}+\sum_{i} \vec{r}^{(i)} \cdot \vec{\sigma}^{(i)}
$$

The goal is again to simulate the evolution due to one specific coupling term $\sigma_{\gamma}^{(l)} \otimes \sigma_{\eta}^{(m)}$. Passing from Eq. (11) to Eq. (2), the first difference is the noncommutivity of the terms in Eq. (2). The second difference is the presence of all three Pauli matrices acting on the same qubit, besides a much larger number of coupling terms.

We adopt the common approach [7, 8, 10, based on the identity

$$
e^{-i K_{1} t_{1}} e^{-i K_{2} t_{2}} \approx e^{-i\left(K_{1} t_{1}+K_{2} t_{2}\right)}+\mathcal{O}\left(t_{1} t_{2}\right),
$$

that employs sufficiently frequent local manipulations (small $t_{i}$ in Eq. (16)) to make the effect of the noncommutivity negligible. With this simplification, the framework in the previous section are readily generalized.

Again, we consider a class of schemes that concatenate (short) equal time intervals of evolution. The essential features for decoupling are as before:

1. In each interval, each $\sigma_{\alpha}^{(i)}$ acquires a + or - sign, which is controlled by the applied local unitaries to be described. 
2. The bilinear coupling $g_{i j \alpha \beta} \sigma_{\alpha}^{(i)} \otimes \sigma_{\beta}^{(j)}$ for $i \neq j$ is unchanged (negated) when the signs of $\sigma_{\alpha}^{(i)}$ and $\sigma_{\beta}^{(j)}$ agree (disagree).

3. To the lowest order in the duration of the time intervals, negating the coupling for exactly half of the intervals cancels it.

The important difference is that, the signs of the three Pauli matrices $\sigma_{\alpha=x, y, z}^{(i)}$ acting on the same qubit $i$ are no longer independent. In fact, the product of their signs has to be + , because $\vec{r}^{(i)}$ in $\vec{r}^{(i)} \cdot \vec{\sigma}^{(i)}$ is transformed by an $\mathrm{SO}(3)$ matrix when local unitaries are applied on the $i$-th qubit. On the other hand, any sign assignment satisfying this constraint can be realized. The possible signs for $\sigma_{x}^{(i)}, \sigma_{y}^{(i)}, \sigma_{z}^{(i)}$ are,,,++++---+---+ , and they can be obtained by applying $I^{(i)}, \sigma_{x}^{(i)}, \sigma_{y}^{(i)}, \sigma_{z}^{(i)}$ respectively before and after the interval. Incorporating these considerations in the previous framework, it can be generalized:

A scheme for $n$ qubits that concatenates $m$ intervals can be specified by three $n \times m$ sign matrices $S_{x}, S_{y}, S_{z}$, related by the entry-wise product $S_{x} * S_{y}=S_{z}$. The $(i, a)$ entry of $S_{\alpha}$ is the sign of $\sigma_{\alpha}^{(i)}$ in the $a$-th time interval.

Note that we omit the number of qubits, $n$, in $S_{x}, S_{y}, S_{z}$ for simplicity. The entry-wise product $* *$ of two matrices is also known as the Schur product or the Hadamard product.

\section{SELECTIVE COUPLING FOR $N$ QUBITS WITH ARBITRARY PAIRWISE COUPLING}

Under the generalized framework, we state the criteria for decoupling and selective coupling for $n$ qubits:

Criteria for decoupling and selective coupling Decoupling is achieved if any two rows taken from $S_{x}, S_{y}, S_{z}$ are orthogonal. Selective coupling of $\sigma_{\gamma}^{(l)} \otimes \sigma_{\eta}^{(m)}$ is achieved if the $l$-th row of $S_{\gamma}$ is identical to the $m$-th row of $S_{\eta}$, but any other pair of rows from $S_{x}, S_{y}$, $S_{z}$ are orthogonal. Local terms are removed if all row sums are zero.

We now using Hadamard matrices to construct sign matrices satisfying these criteria. The first, simpler construction requires $4 \bar{n}$ intervals for $n$-qubits. The second construction improves the requirement to $c 3 \bar{n}$ for $c \approx 1$. In the following, we write $H(\bar{n})$ in place of a decoupling $S_{n}$ for simplicity. This does not affect the argument, extra rows can always be discarded.

To achieve decoupling the sign matrices for the first construction are taken to be

$$
\begin{aligned}
S_{x} & =H(\bar{n}) \otimes[+-+-], \\
S_{y} & =H(\bar{n}) \otimes[++--], \\
S_{z} & =H(\bar{n}) \otimes[+--+] .
\end{aligned}
$$

More explicitly, this construction replaces each entry $\epsilon$ in $H(\bar{n})$ by a row of 4 entries, $[+\epsilon-\epsilon+\epsilon-\epsilon]$ in $S_{x},[+\epsilon+\epsilon-\epsilon-\epsilon]$ in $S_{y}$, and $[+\epsilon-\epsilon-\epsilon+\epsilon]$ in $S_{z}$. The orthogonality conditions can be verified directly. To select the coupling $\sigma_{\gamma}^{(l)} \otimes \sigma_{\eta}^{(m)}$, make the following replacements:
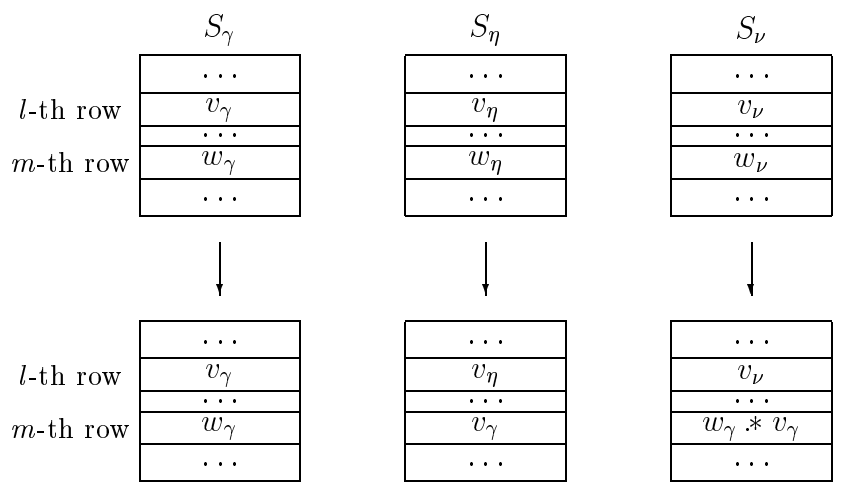

where $\nu \notin\{\gamma, \eta\}$, and $v_{\alpha}, w_{\alpha}$ denote the rows. Now, one has to ensure $w_{\gamma} * v_{\gamma}$ does not appear elsewhere in the 3 matrices. This vector cannot be any one of $v_{\alpha}, w_{\alpha}$. So, the simplest method is to exclude the 3 corresponding rows in $S_{x, y, z}$ that contains $w_{\gamma} * v_{\gamma}$ in the initial choice of $S_{\alpha}$. Note that in both decoupling and selective coupling, all $S_{\alpha}$ have identically zero row sums. Thus in our scheme, local (linear) terms are automatically removed without extra local manipulations. This construction requires $4 \bar{n}$ intervals for decoupling, and $4 \overline{n-1}$ or $4 \bar{n}$ intervals for selective coupling.

We can interpret Eq. (17) as choosing the rows of $S_{x, y, z}$ to be $3 n$ orthogonal rows of the Hadamard matrix $H(\bar{n}) \otimes$ $H(2)^{\otimes 2}$ where

$$
H(2)=\left[\begin{array}{c}
++ \\
+-
\end{array}\right] \text {, and } H(2)^{\otimes 2}=\left[\begin{array}{c}
++++ \\
+-+- \\
++-- \\
+--+
\end{array}\right] .
$$

These $3 n$ rows consist of $n$ disjoint 3 -subsets, the $i$-th one being $\left\{R_{i} \otimes[+-+-], R_{i} \otimes[++--], R_{i} \otimes[+--+]\right\}$ where $R_{i}$ is the $i$-th row of $H(\bar{n})$. The rows in each 3subset entry-wise multiply to $++\cdots+$. Therefore, we can choose the $i$-rows of $S_{x}, S_{y}, S_{z}$ to be the rows of the $i$-th 3-subset, so that $S_{x} * S_{y}=S_{z}$. Note that the rows of a general Hadamard matrix may not form such disjoint 3 -subsets. The particular choice of Hadamard matrix $H(\bar{n}) \otimes H(2)^{\otimes 2}$ satisfies this condition because the last 3 rows in the second tensor component $H(2)^{\otimes 2}$ multiply to ++++ . However, only $3 / 4$ of the available orthogonal rows in $H(\bar{n}) \otimes H(2)^{\otimes 2}$ can be used.

Our second construction avoids this inefficiency by replacing $H(\bar{n}) \otimes H(2)^{\otimes 2}$ with $H\left(\overline{3 n /\left(2^{2 r}-1\right)}\right) \otimes H(2)^{\otimes 2 r}$. 
We prove in Appendix A that for each positive integer $r$, the second through the last rows of the Sylvester matrix $H(2)^{\otimes 2 r}$ form $\left(2^{2 r}-1\right) / 3$ disjoint 3 -subsets, each with rows that entry-wise multiply to $++\cdots+$. The corresponding rows of $S_{x}, S_{y}$, and $S_{z}$ can be choosen to be the tensor products of one row of $H\left(\overline{3 n /\left(2^{2 r}-1\right)}\right)$ with the 3 rows in one such 3 -subset. These rows still have zero row sums by construction. A fraction of $1-2^{-2 r}$ rows can now be used, but the effect of nonexisting Hadamard matrices may be amplified in $H\left(\overline{3 n /\left(2^{2 r}-1\right)}\right)$. One can minimize the required number of intervals $n_{I}$ over $r$, as plotted in Figure 2 for $n \leq 2000$. For large $n, r$ and $n /\left(2^{2 r-2}-1\right)$ can both be large and $n_{I}=c 3 n$ for $c \approx 1$.

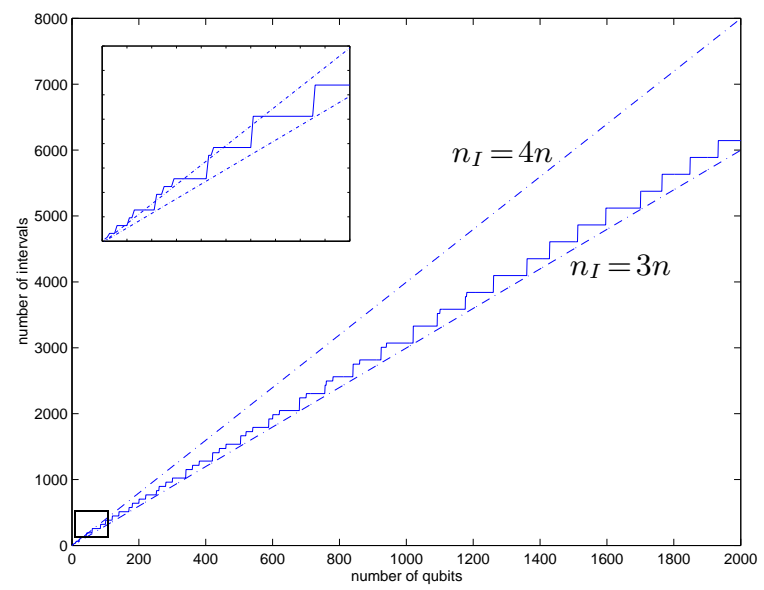

FIG. 2. The plot of the number of interval $n_{I}$ vs the number of qubit $n$ (solid line). The lines $n_{I}=3 n$ and $n_{I}=4 n$ are plotted for comparisons. An enlarged view of the boxed region $(n \leq 100)$ is shown in the inset.

In many applications, one may want to select more than one coupling term from the Hamiltonian. For example, one may select all the coupling terms between the $i$-th and the $j$-th qubits in $H_{2}$. This selection can be made by choosing the $i$-th and the $j$-th rows of $S_{x, y, z}$ to be identical, and any other pairs to be orthogonal. These 6 identical rows have to be $++\cdots+$ in order to satisfy $S_{x} * S_{y}=S_{z}$. The required number of intervals is the same as decoupling $n-2$ qubits. Such selection can reduce the resources to simulate $H_{2}^{\prime}$ with $H_{2}$ [10] by a constant factor.

Comparison The recursive method in Ref. [7] is essentially the particular scheme using $H(2)^{\otimes\left\lceil\log _{2} n\right\rceil}$ in place of $H(\bar{n})$ in Eq. (17).

\section{UNIVERSAL TIME REVERSAL}

We now apply the framework of sign matrices to simulate time reversal. As a first example, we apply the original framework in Ref. [2] to reverse $H_{1}$. First construct a decoupling sign matrix $S_{n}$ for $n$ qubits using a normalized Hadamard matrix $H(\overline{n+1})$, excluding its first row. In this case, all entries in the first column of $S_{n}$ are "+". A sign matrix for time reversal, denoted by $T_{n}$, is obtained by removing the first column of $S_{n}$. In $S_{n}$, any two rows have zero inner product, thus any two rows in $T_{n}$ have inner product -1 , and any coupling term is reversed by the same amount. Furthermore, each row of $S_{n}$ has zero row sum, so, each row in $T_{n}$ has row sum -1 , therefore the local terms are reversed by the same amount as well. Therefore, any Hamiltonian given by Eq. (1) can be reversed. The simulation requires $n_{I}=\overline{n+1}-1$ intervals, and simulates the reversal for the duration of one interval. Hence the simulation factor $\sqrt{10}$ is $n_{I}$. This factor is between $n$ and $n+3$ if Hadamard's conjecture is true, and is $c n$ for $c \approx 1$ in any case. Without local terms, $n_{I}=\bar{n}-1$ ranges between $n-1$ and $n+2$ if Hadamard's conjecture is true. Note that $H_{1}$ can be completely unknown in this protocol.

Comparison It is interesting that our method for any Hamiltonian (even unknown ones) in Eq. (1) achieves a simulation factor very close to the lower bound $n-1$ derived for the much more specific Hamiltonian in Eq. (3) 9].

To simulate the reversal of a Hamiltonian given by Eq. (2), we use the generalization in Section III and IV. The method is exactly the same: First, choose a set of decoupling sign matrices $S_{x}, S_{y}, S_{z}$, using a normalized $H\left(\overline{3 n /\left(2^{2 r}-1\right)}\right) \otimes H(2)^{\otimes r}$ in the second construction (the first row is already excluded in the $S_{x, y, z}$ ). The first columns of $S_{x, y, z}$ contain only + . Removing the first columns results in a reversal scheme. The simulation factor is the same $n_{I}$ as plotted in Figure 2, approaching $3 n$ for large $n$. Again, the protocol applies to any $H_{2}$ and thus applies to unknown Hamiltonian as well.

Comparison The reversal method reported in Ref. [9] for $H_{4}$ in Eq. (4) follows from the method for reversing Eq. (3) when all $d_{\alpha}$ are known and have the same sign, and requires an extra factor of $\frac{\max \left|d_{\alpha}\right|}{\left|d_{x}+d_{y}+d_{z}\right|}$. Thus, the worse case simulation for Eq. (4) is $\approx 3 n$. Surprisingly, our method for the much more general Hamiltonian in Eq. (2) which can be unknown, achieves about the same bound.

\section{CONCLUSION}

We have generalized the framework for Hamiltonian simulation and the methods for decoupling and selective

\footnotetext{
${ }^{5}$ The optimal solution when $d_{\alpha}$ have different signs can be obtained from the method in Ref. [10].
} 
coupling schemes in Ref. [2]. We rederive, as a special case, the crucial step of selecting a coupling term in the simulation of $n$-qubit Hamiltonians in Ref. [7]. We also apply the technique to extend the time reversal problem considered in Ref. [9] from permutation invariant purely nonlocal Hamiltonians to an arbitrary $n$-qubit Hamiltonians.

Our framework based on sign matrices allows the complicated criteria for various simulation tasks to be rephrased in very simple orthogonality conditions, for which solutions can be easily obtained with the connections to Hadamard matrices.

\section{ACKNOWLEDGMENTS}

This generalization was inspired by the work presented in Ref. [7]. We thank Aram Harrow for helpful discussions and suggestions for the paper. DWL is supported in part by the NSA and ARDA under the US Army Research Office, grant DAAG55-98-C-0041.

[1] N. Linden, H. Barjat, R. Carbajo, and R. Freeman, Chemical Physics Letters, 305:28-34, 1999, also arXive e-print quant-ph/9811043.

[2] D. Leung, I. Chuang, F. Yamaguchi, and Y. Yamamoto, Phys. Rev. A, 61:042310, 2000, also arXive e-print quant$\mathrm{ph} / 9904100$.

[3] J. Jones and E. Knill, J. of Mag. Res., 141:322-5, 1999, also arXive e-print quant-ph/9905008.

[4] D. DiVincenzo, Phys. Rev. A. 51, 1015, (1995).

[5] A. Barenco, Proc. R. Soc. Lond. A (1995) 449, 679-83, also arXive e-print quant-ph/9505016, (1995).

[6] D. Deutsch, A. Barenco and A. Ekert, Proc. R. Soc. Lond. A (1995) 449, 669-77, also arXive e-print quant$\mathrm{ph} / 9505018$, (1995).

[7] J.L. Dodd, M.A. Nielsen, M.J. Bremner, and R. T. Thew, arXive e-print quant-ph/0106064

[8] P. Wocjan, D. Janzing, and Th. Beth, arXive e-print quant-ph/0106077.

[9] D. Janzing, P. Wocjan, and Th. Beth, arXive e-print quant-ph/0106085v1.

[10] C. H. Bennett, J. I. Cirac, M. S. Leifer, D. W. Leung, N. Linden, S. Popescu, G. Vidal, arXive e-print quant$\mathrm{ph} / 0107035$.

[11] C. Colbourn and J. Dinitz (Eds) The CRC Handbook of Combinatorial Designs (CRC Press, Boca Raton, 1996).

[12] http://www.research.att.com/ njas/hadamard/index.ht$\mathrm{ml}$

[13] J. van Lint and R. Wilson, A Course in Combinatorics (Cambridge University Press, Cambridge, 1992).

[14] F. MacWilliams and N. Sloane, The theory of ErrorCorrecting Codes (North Holland, Amsterdam, 1977).
[15] J. Hadamard, Bull. Sciences Math., (2) 17 (1893), 240246.

[16] J. Sylvester, Phil. Mag. 34 (1867), 461-475.

[17] R.E.A.C Paley, J. Math. Phys. 12 (1933), 311-320.

\section{APPENDIX A: SYLVESTER MATRIX}

In this appendix, we show that the $2^{2 r}-1$ rows (the 2 nd through the last rows) of the Sylvester matrix $H(2)^{\otimes 2 r}$ partition into disjoint 3 -subsets, each with 3 rows entrywise multiply to $++\cdots+$.

Consider $H(2)=\left[\begin{array}{rr}1 & 1 \\ 1 & -1\end{array}\right]$. We label the rows and columns by 0 and 1 . For example, the $(0,0)$ entry of $H(2)$ is 1 . We use the shorthand $H_{i j}$ for the $(i, j)$ entry of $H(2)$. Note that $H_{i j}=(-1)^{i j}$. For $H(2)^{n}$, one can label the rows and columns with the composite index $\mathbf{i}=\left(i_{1}, i_{2}, \cdots, i_{n}\right)$ which is $n$-bit long. In particular,

$$
\begin{aligned}
H_{\mathbf{i j}} & =H_{i_{1} j_{1}} \times H_{i_{2} j_{2}} \times \cdots \times H_{i_{n} j_{n}} \\
& =(-1)^{i_{1} j_{1}+i_{2} j_{2}+\cdots+i_{n} j_{n}} \\
& =(-1)^{\mathbf{i} \cdot \mathbf{j}}
\end{aligned}
$$

where $\mathbf{i} \cdot \mathbf{j}$ denotes the usual inner product of $\mathbf{i}$ and $\mathbf{j}$. Claim: The $\mathbf{i}$-th, $\mathbf{j}$-th, $\mathbf{k}$-th rows entry-wise multiply to $++\cdots+$ if and only if $\mathbf{i} \oplus \mathbf{j} \oplus \mathbf{k}=\mathbf{0}$ where $\oplus$ is the bitwise additional modulo 2 , and $\mathbf{0}$ is an $n$-bit string of all 0 .

Proof: For each column l,

$$
H_{\mathbf{i l}} \times H_{\mathbf{j} \mathbf{1}} \times H_{\mathbf{k} \mathbf{l}}=(-1)^{\mathbf{i} \cdot \mathbf{l}+\mathbf{j} \cdot \mathbf{l}+\mathbf{k} \cdot \mathbf{l}}=(-1)^{(\mathbf{i}+\mathbf{j}+\mathbf{k}) \cdot \mathbf{l}}
$$

The $\mathbf{i}$-th, $\mathbf{j}$-th, $\mathbf{k}$-th rows entry-wise multiply to $++\cdots+$ iff $\forall_{\mathbf{l}} H_{\mathbf{i} \mathbf{l}} \times H_{\mathbf{j} \mathbf{l}} \times H_{\mathbf{k} \mathbf{l}}=1$ iff $\mathbf{i} \oplus \mathbf{j} \oplus \mathbf{k}=\mathbf{0}$. Hence, the problem reduces to showing that, the set of nonzero $n=2 r$-bit strings partitions into $\left(2^{2 r}-1\right) 3$-subsets, each with 3 elements bitwise sum to 0 . This can be proved by induction on $r$. 